\title{
IPv6 R\&D Activities in WIDE Project
}

\author{
Hiroshi Esaki \\ The University of Tokyo, \\ 7-3-1 Hongo, Bunkyo-ku, \\ Tokyo, 113-8656, Japan \\ hiroshi@wide.ad.jp,
}

\author{
Akira Kato \\ The University of Tokyo, \\ 2-11-16 Yayoi, Bunkyo-ku, \\ Tokyo, 113-8658, Japan \\ kato@wide.ad.jp,
}

\author{
Jun Murai \\ Keio University \\ 5322 Endo, Fujisawa-shi, \\ Kanagawa, 252-8520, Japan \\ junsec@wide.ad.jp
}

\begin{abstract}
The WIDE project, www.wide.ad.jp, is a research consortium among industry and academia. WIDE project is consist of more than four hundred active researchers from more than hundred private companies and from more than forty universities. The WIDE project operates the nation-wide R\&D testbed, which is connected with many other R\&D testbeds, and the NSPIXPs, that are the largest IXes in Japan. The main focus of WIDE project has been IPv6 technology and has established IPv6 testbed since 1998. Basically, all of R\&D activities in WIDE project are based on the IPv6. This paper describes the overview of WIDE project's IPv6 R\&D activities, such as KAME / USAGI / TAHI / Nautikus6 projects.
\end{abstract}

\section{Introduction}

The Internet technology provides the global and ubiquitous digital communication platform using the various types of datalinks. At the first stage, the Internet had been established and operated for the researchers and scientists using the computer technology. Through the deployment of Internet technology to the industrial activities and people's daily life, the Internet technology has mutated to a common and essential infrastructure and platform for all of people, including peoples who would not be familiar with the computer technology. The digital infrastructure assumes the IP technology as the core and common platform, which flexibly adopts various needs required by the various digital media and applications. To achieve goal, the Internet must continue the mutation and revolution through the invention and the introduction of new technologies. In order to develop the "reality" of these new technologies, we need the following four steps.

1. Standardization

2. Referenced implementations

3. Interoperability and conformance of products

4. Professional operation on the live network

It is very important to build and operate a practical live testbed, which accommodates the traffic generated by various users (i.e., such as researchers, engineers and ordinary users). On this testbed, the researchers and engineers can evaluate and validate the new technologies invented and developed by themselves. Through this practical operation, the technical issues will be newly realized and recognized, so as to work on the new research and development items. This is a positive spiral among the testbed and R\&D.

Since 1998, the WIDE project has established the full scale nation-wide IPv6 testbed, cooperating with the global $\mathrm{R} \& \mathrm{D}$ networks, in order to accelerate and integrate the R\&D activities on the IPv6 technology. On the WIDE IPv6 network, the operating system, middleware and applications developed by the researchers in the WIDE project with the industries has been evaluated and has been widely used by various research projects and industry in the global internet community. This paper describes the updated overview of the WIDE IPv6 projects from [1].

\section{WIDE IPv6 Project}

\subsection{Structure of WIDE IPv6 tested}

The WIDE IPv6 testbed must be a part of global IPv6 network. The testbed has been interconnected with various other R\&D network, e.g., APAN, APII, AI3, Abilene, GLIF/IEEAF. Domestically, the WIDE IPv6 testbed as been tightly collaborate with the ITRC (Internet Technology Research Consortium) and the CKP (Cyber Kansai Project). The WIDE IPv6 testbed has been overlaid on the following platforms.

- JGN II (Japan Gigabit Network, www.jgn.nict.go.jp)

- APII testbed (www.apii.net/)

- APAN/TransPac (www.apan.net/)

- AI3 testbed (www.ai3.net/)

- GLIF/IEEAF (www.glif.is/)

\subsection{R\&D activities on the Platform Technology}

\subsubsection{IPv6 Core Technology}

(1) IPv6 Protocol Stack

WIDE project has been progressed IPv6 R\&D activities since middle of 1990's. Especially, the following three core project has been established.

- KAME project (www.kame.net) for BSD Unix

- USAGI project (www.linux-ipv6.org/) for Linux

- TAHI project (www.tahi.org/) for evaluation and test suit against IPv6 products

TAHI project has hosted many interoperability test events, and has been cooperated with PLUG group of ETSI and Connectathon. Since the beginning of 2003, the extended TAHI team, which is the Certification WG of IPv6 Promotion Council (www.v6pc.jp), has been participated in the IPv6 Ready Logo Program run by IPv6 Forum (www.ipv6forum.com), and has played an important role for this program, collaborating with UNH-IOL (www.iol.unh.edu) and IRISA (www.irisa.fr).

(2) DNS System

DNS system is a global scale distributed directory service between IP address and FQDN (Fully Qualified Domain Name). As for DNS system, we have following two projects. One is the design and operation of thirteen root DNS servers, collaborating with USC-ISI (www.isi.edu) and ISC ((Internet Software Consortium, www.isc.org). Here, WIDE project has the responsibility on the operation of $M$ route server. The other project is the development of "bind" for IPv6 with ISC. 


\subsubsection{DVTS (http://www.sfc.wide.ad.jp/dvts/)}

Digital Video (DV) is a high quality video image compression format for TV quality. DV uses an intra-frame compression for video signal, and uses an 12 bit or 16 bits PCM for sound signal. From this point of view, the DV signal would be tolerant for packet losses regarding the video image transmission, comparing with the video transmission using the other compression mechanism (e.g., MPEG2). We have developed the DVTS (Digital Video Transmission System), that encapsulates the DV signal via IEEE1394 interface into the IP packets to transmit over the Internet[2]. DVTS runs with RTP (Real-time Transmission Protocol) both over IPv4 and IPv6 protocol stack. DVTS consumes about 35Mbps bandwidth. However, when the available bandwidth between source and destination nodes are not enough large, the video frame can be periodically discarded at the sender node to reduce the required bandwidth for DV transmission. DVTS runs over various platforms, e.g., BSD-UNIX and Linux with X-window system, Windows with direct-X and Macintosh.

Since the beginning of 2004, WIDE project has collaborating with Internet2 community to develop and deploy the DVTS system over Internet2 network.

\subsubsection{Large Scale Multicast using PIM}

The first large scale PIM-SM operation on WIDE project testbed was on November 27 in 1999. The technical conference held at Kurashiki University of Science and Arts had struggled to be multicasted toward more than ten sites in nation-wide Japan, using the DV (Digital Video) stream. We have realized a lot of technical issues. In March of 2002, the interactive multicast remote conference among USA and Japan, collaborating by Internet2/Abeline, FLA (Fujitsu Lab. America) in Maryland, NTT Communication MCL in Palo Alto and WIDE project. In January of 2005, we have operated large scale DV

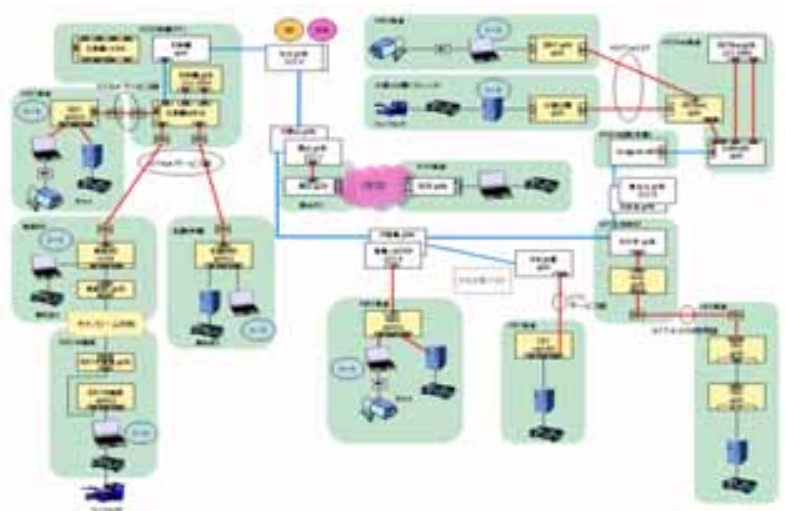

multicasting using PIM-SSM (Source Specific Multicast), over nation-wide JGN2 network.

Fig. 1 PIM-SSM Operation over JGN2

\subsubsection{Mobile Technology}

We have initiated the network mobility, called as NEMO (NEtwork MObility) work at the IETF, collaborating with INRIA, in 2003. The architecture is an extension and modification of mobile IP. We have established nautilus6 project (www.nautilus6.org) and SHISA project (www.mobileip.jp). One of the major applications using these research activities would be the InternetCAR project, described below.

Based on the discussion in WIDE project, we have realized that these mobile technologies are not only available for mobile nodes or mobile networks, but are also available for wired networks [3]. Mobile networks could be a superset of fixed networks. Mobile technologies can provide the following functions, which are required for wired network, as well.

1. Multi-homing ; Connecting the network via multiple links. Multiple links could be used for achieving higher robustness, load balancing or policy routing.

2. Plug-and-play; When the network changes physical or logical location, the network prefix of the network is generally changed. By applying the NEMO technology to a campus network, the campus network is not required renumbering nor system reconfiguration, when it changes the upstream ISP.

\subsubsection{UDLR (Uni-Directional Link Routing) [4]}

The routing protocol running in the Internet assumes that the link is bi-directional and symmetric. However, when we look at satellite link, it is uni-directional. We have discussed the UDLR technology at the IETF collaborating with INRIA, since 1997. Also, we have established the satellite internet testbed using the UDLR technology. The testbed is called as AI3(www.ai3.net/), that is collaborating with NiCT/CRL (www.nict.go.jp) and JSAT (www.iijnet.or.jp/JSAT/). The AI3 network has covered the Asian countries as shown in Fig.2. The new research item on UDLR system is IPv6 multicasting, and dynamic bandwidth allocation with production quality operation.

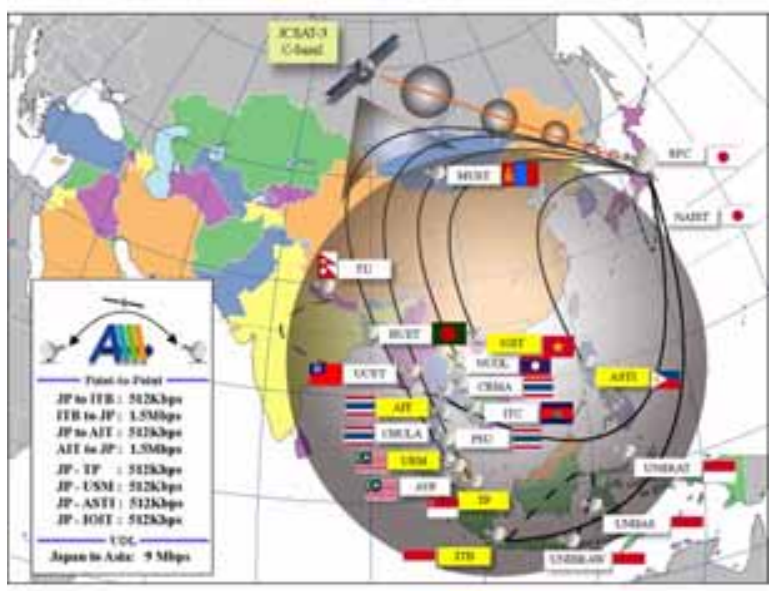

Fig.2 AI3 Service Footprint

\subsection{Applications of IPv6 Technologies}

After the application of IPv6 technology to legacy networks, such as ISP or R\&D networks, we are exploring new application area. These area are focusing on reducing system and operational cost and on improving operational efficiency. We realize that, when we have a lot of sensors or controllers internetworked by IP, the digital information 
will be able to contribute to the reduction of system cost and to the improvement of human activities and systems.

\subsubsection{InternetCAR (www.sfc.wide.ad.jp/InternetCAR/)}

InternetCAR Project is connecting the automobiles, which has the autonomous internet node function, to the internet. Nodes in the automobile can be accessed from the Internet and can access the Internet. We have operated several testbed using real automobiles (e.g., taxi or bus), in large metropolitans, such as Yokohama or Nagoya. Some applications, such as a remote sensing using the speed mater and wiper's position, has been operated and evaluated. The testbed system adopts the mobile technologies, such as Mobile IPv6, NEMO (Network in Motion), dynamic tunneling or vertical hand-over. Figure 3 shows the overview of the demonstration system using the vertical hand-over with NEMO in Nagoya City in 2004.

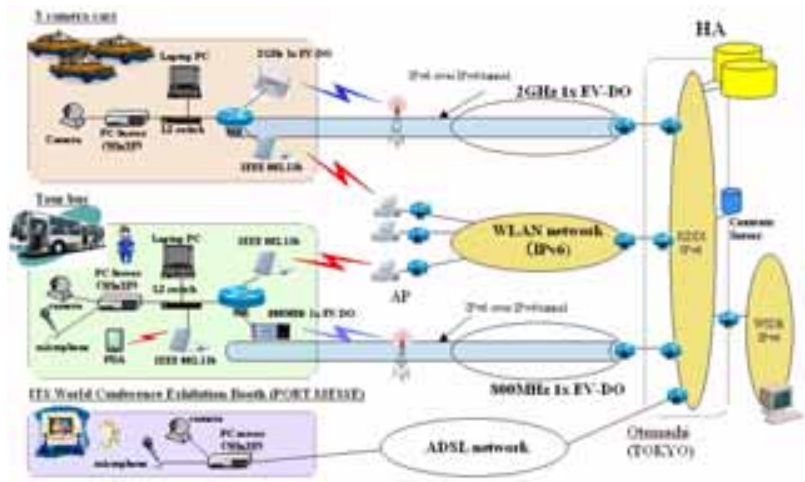

Fig.3 NEMO demonstration in Nagoya City in 2004.

\subsubsection{SOI (School of Internet) Project}

The virtual university, SOI, has been established on the Internet since 1997. The students of SOI has participated in through various network platform. The SOI testbed has been across the Asian Pacific countries. SOI operated two studios in USA, i.e., FLA (Fujitsu Lab. America) in Maryland and NTT Communications MCL in Palo Alto, and is covering the Asian Pacific countries via satellite link collaborating with AI3 project. This SOI project collaborating Asian-Pacific area is called as SOI-AP (SOI Asian Pacific) Project.

\subsubsection{Facility Networking}

Facility networking includes factory automation, building automation or home automation, which is the integration of different type of networks, such as sensor network, control network or security network. The facility networking would be toward the integration of real-space and cyber-space using common IP technology.

\section{(1) Building Automation}

We are working with the industry so that the IPv6 is applied to the basic protocol to be shared by various systems in building automation. Since they have used proprietary technologies for each company and for each sub-systems, they are interested in the IP-Centric system, to achieve highly effective system operation. We had the world first interoperability testing among building automation components with IPv6 transport, using the BACnet[5] and LonWorks[6], in 2004.

(2) Live E! Project (www.live-e.org)

Live E! Project is a consortium founded by WIDE project and IPv6 Promotion Council. This project is aiming to establish the platform to share all the digital information, generated by any digital devices. These digital devices are installed and operated by individual and by organizations. The information should be related with the live environmental information of the Earth. By sharing this digital information, we will be able to create new applications, which can contribute to safety and effective space(environment. Some applications, such as basic information for the protection of environment (e.g., a heat-island phenomena in metropolitan), educational material, public service, public safety or business applications, could use the common digital information for different purposes. The related original work would be back in 2001, when the WIDE project with Yokogawa Electric Corp.(www.yokogawa.com) had developed a temperature node with IPv6 protocol stack, called i-node(www.i-node.co.jp).

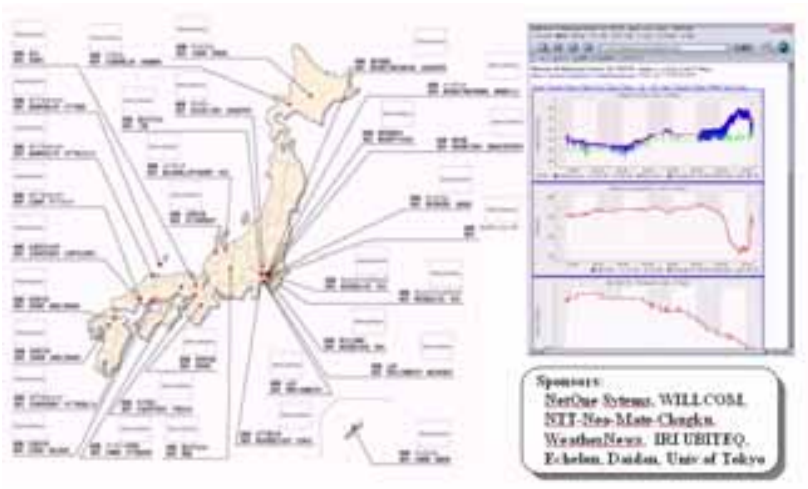

Fig.4 Installation of weather stations across Japan

\subsubsection{IPv6 Transition Trials}

WIDE Project has been worked with Internet Association Japan (IAJapan, www.iajapan.org), IPv6 Promotion Council and NiCT/MIC for recent three years on IPv6 transition trials. The trial aims the professional IPv6 transition from the existing operation networks, which includes SOHO, corporate campus network, local government networks and home network.

\subsubsection{IPv6 Fix (http://v6fix.net/)}

Because of small and minor technical problems or mis-configurations, many system operators may want to turn off IPv6 function, for conservative operation of their networks. We recognized some inappropriate technical description and specification in some RFCs, some inappropriate implementations, or some misunderstandings. The purpose of IPv6 Fix project is to fix this situation and to share the operational knowledge on IPv6 operation.

\subsubsection{Security model for IPv6 System}

secure6 working group identifies the security problems and challenges on IPv6 network. With IPv6, it would be a 
good opportunity to re-consider the best/better security architecture for the network accommodating huge number of computers and of non-computer appliances without NAT technology. The working group researches new protocols and proposes it as a standard, releases a reference implementation to deliver new security model (post firewall model) which is well adapted for IPv6 network.

\section{WIDE Project Network Configuration}

The WIDE backbone network consists of various speed of links from 2Mbps CBR ATM upto 10Gbps Ethernet. Some of these links are provided by JGN2, that is an VLAN/Ethernet based nation-wide R\&D network run by NiCT. Most of the network is IPv4/IPv6 dual stack operation. WIDE backbone is multi-vendor network including such as Cisco, Foundry, Juniper, and Hitachi. PCs running NetBSD with Zebra routing daemon are also used mainly for IPv6 routing especially for IPv6 multicast.

Figures 5 and 6 shows the network topology of WIDE backbone.
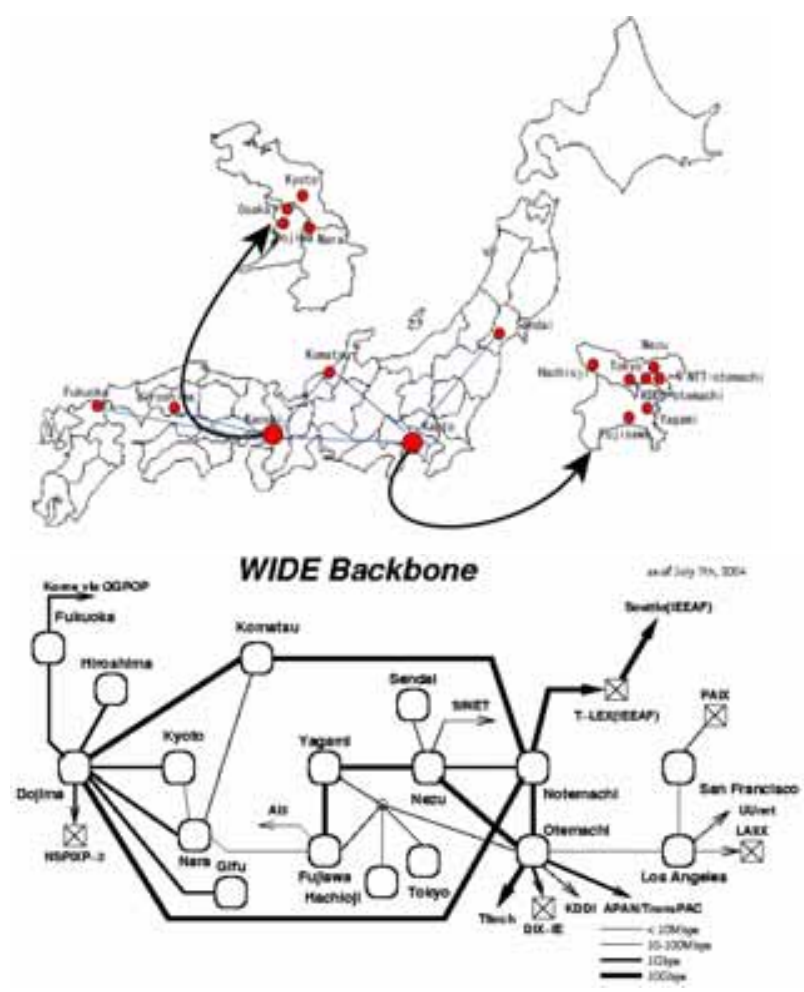

Fig.5 Geographical view of WIDE Backbone

WIDE backbone has various external connectivities. For example, WIDE internet is participating to APAN and is connecting with an GbE interface. As for our native link to United States, we had an OC-3 extention from Los Angeles to San Jose, in order to have an access to PAIX with Fast Ethernet.

WIDE project has run the DIX-IE (or former name was
NSPIXP-2) in Tokyo and the NSPIXP-3 in Osaka respectively. Though it is not shown in the figxxx, WIDE backbone also has run and has been connected to the NSPIXP-6, which is a purely experimental but is one of the largest pure IPv6 IX accommodating about 50 ISPes in Japan or from oversea.

WIDE project has established the T-LEX, called as Tokyo Lambda Exchange point. T-LEX is yet another L1/L2/L3 academic exchange point, that is especially focusing on the global lambda networking research.

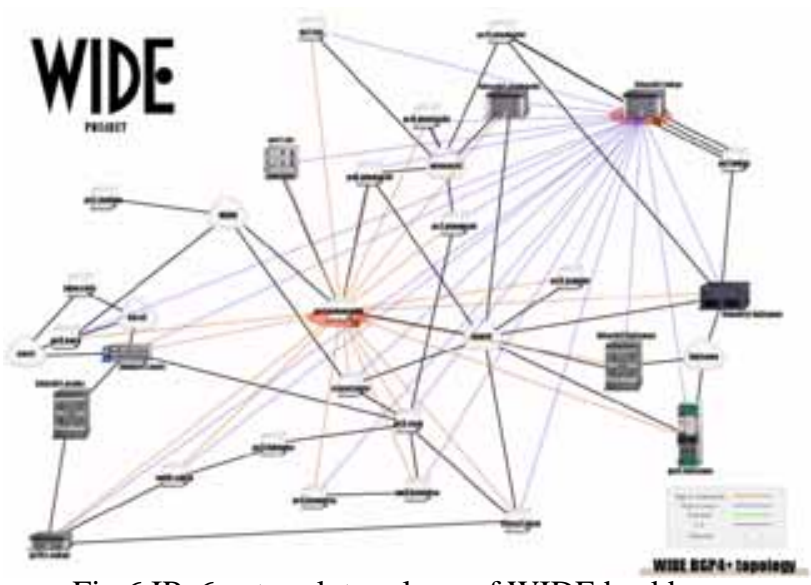

Fig.6 IPv6 network topology of WIDE backbone

\section{Conclusion}

WIDE project is a research forum among industry and academia regarding the internet technology, covering wide variety of research area. Almost all of $R \& D$ activities assume the IPv6 technology as a common platform as layer 3 protocol. The R\&D are on platform technologies, middleware technologies, applications and operational technologies. WIDE project operates the $R \& D$ testbed, which uses state-of-art technologies, in order to evaluate the new technologies and to realize the further R\&D items through the practical operation.

\section{Acknowledgement}

WIDE internet operates collaboration with and contribution from various organizations, such as NiCT JGN2 and APII testbed, AI3, PoweredCom/TTnet, NTT Group, GAL, Cisco Systems or Hitachi/AlaxalaNetworks.

\section{References}

[1] H.Esaki, A.Kato, J.Murai, "R\&D Activities and Testbed Operation in WIDE Project", Workshop on IPv6 and Applications at IEEE/IPSJ SAINT2003, Orlando Florida USA, January 2003.

[2] K.Kobayashi, A.Ogawa, S.Casner, C.Bormann, "RTP Payload Format for DV(IEC 61834) Vieeo”, IETF RFC3189, Jan.2002.

[3] H.Esaki, "Multi-Homing and Multi-Path Architecture Using Mobile IP and NEMO Framework", Panel 1 (The Future of the Internet Architecture) at IEEE/IPSJ SAINT2004, Tokyo, Japan, January 2004.

[4] Home Page of UDLR, http://www.udcast.com/udlr/

[5] Home Page of BACnet, http://www.bacnet.org/

[6] Home Page of Lon Mark, http://www.lonmark.org/ 